Speichelfluss fast ganz verloren, am auffallendsten aber hat sich die Stimme, die viel klarer und lauter ist, gebessert, selbst das Schlucken ist etwas freier, sodass der Kranke geriebenes Fleisch, auch Brod mit viel Flüssigkeit hinabspülen kann. Auch hat sich der Kräfte\%ustand gebessert, sodass Patient wieder allein gehen kann. Die Arsenikkur gedenke ich noch länger fortzuset\%en, um dadurch die Verkleinerung der offenbar vorhandenen Schlundgeschwulst herbeizuführen, wozu Aussicht vorhanden scheint, und damit der Inanition des Alten entgegenzutreten und seine lebensdauer zu verlängern.

\title{
Litteratur.
}

Mikulič, J., Ueber eine eigenartige symmetrische Erkrankung der Thränenund Mundspeicheldrüsen (Beitrïge \%ur Chirurgie, Festschrift, gewidmet Th. Billroth etc. Stuttgart 189\%. S. 610-630.)

Kümmel, Werner, Weitere Beitrïge zur Lelhre von der symmetrischen Eirkrankung der Thrinen- und Mundspeicheldrūsen (Mikulic\%). - (Zusammenstellung von 21 früheren Fällen und 6 nenen eigenen. Mitteilungen aus den Grenzgebieten der Medizin u. Chirurg. Bd. 11. S. 111-141. Jen:i 1897.)

Hochheim, W., Ein Beitrag \%ur lienntnis der symmetrischen Lid- und Orbitaltumoren (Graefe's Archiv. 31. Bd. S. 347-379.)

Wingen roth, Ernst, Ein Beitrag \%ur Kenntnis der symmetrischen Tumoren der Augenlider (ebenda, S. 380-390).

III.

\section{Ein weiterer Fall von in den Glaskörper vordringender Arterienschlinge.}

Von

\author{
Dr. I. AI.EX A NDER, \\ Augenarzt in Nürnberg.
}

In den Ḱlinischen Monatsblättern für Augenheilkunde, Maiheft 1899, hat Herr Kollege Günsburg') in Breslau einen Fall von in den Glaskörper vordringender Arterienschlinge beschrieben und durch beigefügte Skizze näher veranschaulicht. Bei seinen Litteraturstudien kam er zu dem Ergebnis, dass sein Fall gleichsam ein Unikum wïre; ïhnliche Bilder konnte er auch nur in drei Mitteilungen wiederfinden, $\mathbf{u}$. a. in der Beobachtung von Hirschberg, welcher derartige Abnormitäten im Gefüssrerlauf als nunerhört selten" bezeichnete. Da seit der Günsburg'schen Arbeit 
hierüber nur wenig mehr bekannt geworden ist, dürfte folgende Beschreibung von einigem Interesse sein.

Der 47 jährige Bäckermeister L. kam im lebruar 1903 wegen katarrlıaischer Beschwerden und, um sich ein Leseglas verordnen z." lassen, in. meine Sprechstunde. Das linke Auge ist rollkommen normal, speriell zeigt die Anordnung der Net\%hatgefïsse nichts Abnormes. S (-0,5 cyl - $6 / 10$

Dagegen ergiebt dic ophthalmoskopische C̈ntersuchung des rechten Auges folgendes interessante Bild:

Aus der Gefïsspforte herausgetreten, teilt sich die A. centr. retinac in einen oberen und einen unteren $\lambda$ st. Der obere Ast zeigt in seiner Gefāssser\%weigung nichts besonderes; der untere dagegen bietet dic genannte merkwärdige Anomalie dar. Anf der Selınervenscheibe teilt sich der untere Ast in dic $A$. tenup. inf. und in dic $A$. nas. infer. Let\%tere lïuft nun nicht direkt zu ihrem Ver\%weigungsbe\%irk hin, sondern - unmittelbar nach ibrer Trennung, also noch anf der Papille - dringt sie erst in sagittaler Richtung in den Glaskürper ror, um dann nach vorn unten \%u verlaulen. Plöt\%lich aber biegt sie zu einer. Schlinge um, und, inden sie das bisherige Gefäss. rohr in 4-j Windungen umläuft, gelangt sie \% ihrer Ursprungsstelle \%.1. rück, um dann in normaler Weise nach unten $\%$ riehen und sich in ihrem Netzhautbezirke aus\%ubreiten.

\%wischen der Schnervenscheibe und der liuppe der Arterienschlinge besteht eine Jinstellungsdifferen\% von 5 Dioptrien: die Schlinge ist demnach $12 / 3$ min lang.

Die liarbe des Windungsstückes ist erheblich dunkler als die des Achsenstückes. Es mag dahin gestellt bleiben, ob dieser Unterschied nur cine Reflexwirkung ist oder ob cr bedingt wird dureh Cirkulationsstörungen in mehr oder weniger behinderter P'nssage. Die farbendifferen\% ist so benerkenswert, dass man das zour Papille zurück laufende Gefïss für eine Vene balten könute, wäre das Vorkommen einer solehen anatomisch sicher gestellt. Das ror- und rückisirts strebende

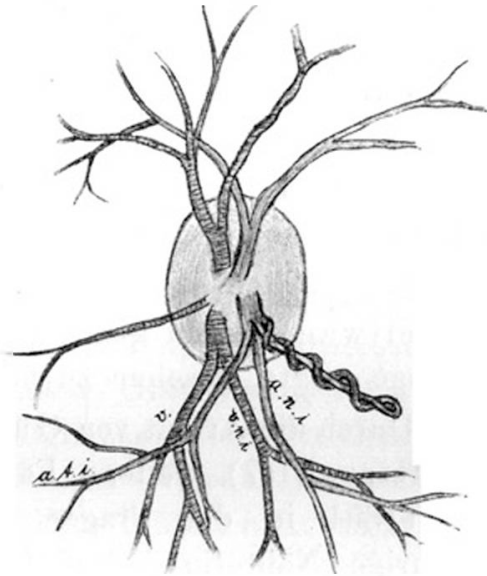

R. (aufrechtes Bild).

Arterienschlinge in den Glaskörper rordringend. Stīck ist ron einer \%arten Biudegewebsscheide umgeben, die sich auf der Papille verliert; nuf diese Weise erinnert die Gefïsschlinge noch mehr an die ron der Amnionscheide umschlossene Nabelschnur.

Stellt man genau auf die Kuppe der Scllinge ein ( $+j$ Dioptr.), so kann man keine Bewegung derselben wahrnehnen. Sobald aber nun ein geringer Druck auf den Bulbus ausgeübt wird, überzeugt man sich leicht, dass der wanze abnorme Gefässabschnitt Oscillationen macht, die synchron dem Radialpulse sind; dabei bewegt sich der Stamm ron rechls nach links und umgekichrt, wahrend die Spit\%e der Schlinge ron oben nach unten sich senkt resp. vou unten nach obeu sich hebt, also Nickbewegungen atusführt.

Auch beim stārksten, d. h. noch rom Auge geduldeten Drucke war ein Erblassen der Gefaissschlinge nicht \%u erziclen; es wurden die genannten Bewegungen in ihrer Exkursion nur intensiver. 
Von der beschriebenen Abnormităt abgesehen, bot der Augenhintergrund (auch das übrige Auge) nichts besondores. $\mathrm{S}=6 / 8 \mathrm{E}$. ist normal.

Eutoptische Walhrnehmungen wurden nicht gemacht. Das Gesichtsfeld

Es handelt sich im vorliegenden Falle, ebenso wie in dem von Günsburg beschriebenen um eine frei in den normalen Glaskörper hineindringende Arterienschlinge. Von den früher publizierten Beobachtungen lässt $\mathrm{G}$ üns burg nur die von Czermik (2), Hirschberg (3) und Wachtler (4) gelten, während die übrigen fast ausschliesslich zu den Fiallen von Art. hyaloidea persistens zu rechnen sind.

$\mathrm{Zu}$ der uns interessierenden Gruppe gehört nach Wintersteiner (כ) auch ein (von Günsburg übersehener) Fall ron Bock, der nallerdings als unzweifelhafte Arteria hyaloidea persistens bei einem 21 jährigen Mann mit normaler Sehschärfe bezeichnet wurle; allein die Beschreibung, nabelschnurartig gewundenes Gefäss", das aus dem Gefässtrichter in den Glaskörper vordrang und vorn mit keulenförmigem Ende umbog und wieder \%ur Papille zurückkehrte, lässt keinen Zweifel, dass es sich um die gleiche Anomalie handelte. Diese Schlinge war ca. $10 \mathrm{~mm}$ weit in den Innenraum des Auges zu verfolgen".

Wintersteiner selbst erinnert sich in den Spiegelkursen v. Stellwag's einen gleichen Fall, der auch starke Pulsation der Schlinge zeigte, gesehen \%u haben.

Durch die Arbeit von Günsburg angeregt, laaben Bondi (6) und Hirsch (7) weitere Fälle veröffentlicht. Bondi's erster Fall (1894 in der Prager deutschen Augenklinik), der eine 18 jährige Näliterin betraf (nur am rechten Auge), besitzt eine so frappante Aehnlichkeit mit dem Giinsburg'schen, dass man „bei nur oberflächlicher Vergleichung beiler Zeichnungen an eine ldentität dieser Fälle denken muss". Dic Läinge der Schlinge betrug hier ca. $1 \mathrm{~mm}$.

In Bondi's zweitem Falle bildete die Arteric nur eine einfache Schlinge, es fehlten die Windungen um die Lïngsachse.

Hirsch berichtet über eine Arterienschlinge, die über 2,3 $\mathrm{mm}$ lang ist. Sein Fall ist - abgesehen von der Grösse der Gefüssanomalie - auch insofern besonders bemerkenswert, als hier die Schlingenbildung ausserordentlich ïbersichtlich ist. „Es krümmt sich die untere Hauptarterie, nachlem sie gleich der oberen aus der normal gelagerten arteriellen Gefässpforte ausgetreten, häkchenfürmig um und erhebt sich sogleich aus dem Papillenniveau, um fast genau senkrecht zur Fläche des Seh- 
nervenkopfes gegen die Mitte des Glaskörpers zu laufen, und nachdem sie sich hier in gan\% eigentümlicher Weise zu einem Knoten viermal umschlungen, kehrt sie parallel mit sich selbst zur Papillenebene \%urück und zwar genau wieder \%u dem Punkte der Unterbrechung ihres papilliiren Verlaufes." Besonders deutlich ausgeprägt waren hier die pulsatorischen Bewegungen, die in unserem Falle erst bei Fingerdruck auf dias duge zu erzielen waren.

Der Befund war auch hier ein rein zuffilliger; der 21 jührige Patient hatte auf dem betreffenden Auge (linken) mit $-1,5 \mathrm{D}$ $S=n m_{3}$ !

In den bisher bekannten Fällen verursachte die Gefäissanomalie keine Sehstorung. Eine praktische Bedeutung scheint daher dieser $\Lambda$ bnormitit, wie auch Bondi bemerkt, nicht zu\%ukommen. Die Möglichkeit ist allerdings nicht von der Hand $\%$ weisen, dass solche Arterienschlinge auch cinmal - durch spontane Ruptur oder durch traumatische Verletzung - die Quelle einer intraocularen Blutung mit deren event. perniciösen Folgen werden kann, ähnlich wie es bei noch bluthaltiger Arteria hyaloidea persistens schon rorgekommen ist.

Auf welche Weise das interessante Bild zustande kommt, ist noch nicht bestimmt \%u sigen. Čermak glaubt sicher, dass „hier der hest einer jener embryonalen Gefaissarkaden vorliegt, wie sie durch die mannigfaltige Verïstelung, Anastomosierung und Sprossenbildung der embryonalen Glaskörpergefüsse entstehen“. Er hält aber auch die Möglichkeit der Beziehung zur Art. hyaloidea nicht für ausgeschlossen. Wachtler denkt an eine Be\%iehung \%u den Vasa hyaloidea Kölliker's, lässt aber auch die Annahme von als Schlingenbildung auftretenden Verlaufsamomalien der Netzhautgefässe selbst" bestehen.

Vinen Zusammenhang mit der Arteria hyaloidea persistens, den einige Autoren angenommen haben, weist ( $\mathrm{i}$ ïnslourg entschieden zurück; schon v. Michel (8) hat vorher in seinem Jehlıbuche, in welchem er in dem Kapitel der angeborenen Gilaskörperabweichungen auch der Gefüssschlingen gedenkt, diese Beziehung bestimmt in Abrede gestellt.

In let\%ter \%eit hat noch einmal v. Hippel(9) betont, dass die genannten Arterienschlingen nichts mit der Arteria hyaloidea \%u thun haben.

Der zur Papille zurückstrebende T'eil des Crefïisses ist wiederholentlich als eine Vene, ron den dutoren, die einen Zusammenhang der Gefüssschlinge mit der Art hyaloidea amnahmen, direkt als Vena hyaloidea bezeichnet worden. 
Liebreich (10) \%. B. hat seinen Fall in diesem Simne gedeutet.

Auch bei unserem Patienten konnte man leicht in Versuchung kommen, den um die Längsachse gewunclenen und \%ur Papille zurückkehrenden Gefässabschnitt für renöser Natur «u halten. Es bestand, wie schon oben herrorgehoben wurde, eine bemerkenswerte Differen\% in der Farbe beider Gefüsspartien.

Die Annahme, es könnte sich hier event. um eine Vena hyaloidea handeln, ist aber von der Hand zu weisen, da eine Glaskörpervene bisher noch nicht im menschlichen Ange festgestellt worden ist. [Schapringer (11), Becker (12), r. Michel, v. Hippel u. a.]

Meines Frachtens ist die geschilderte Abnormitit in Parrallele z.u set\%en mit der Schlingenbildung der Gefiisse im Niveau der Netzhaut selbst, eine Ansicht, die auch ron Hirsch ausgesprochen wurde. Bis auf die Niveandifferen\% wäre \%. B. der von Szili (13) mitgeteilte Fall einer merkwürligen Schlinge der Net\%hautschlagader in dem rechten Ange eines 19 jährigen Mannes mit dem unsrigen identisch.

Die in den Glaskörper vordringende Arterienschlinge wäre demnach nur als eine Steigerung einer Net\%hautgefüssschlingelung anzusprechen.

\section{Litteratur.}

1. Günsburg, le, lïn fiall von in den Glaskïrper rurdringender Arterienschlinge. Klin. Monatsblätter für Augrenheilk. 1899. S. 173.

2. Crermak, W., Ein liall einer in den Glaskürper vordringenden arteriellen Gefässschlinge und Sehnerrenausbreitung. Hirschberg's Centralblatt. 1883. S. 289.

3. Hirschberg, Ein l'all von praepapill. Gefāssschlinge der Netzhautschlagader. Centralbl. 1885. S. 205.

4. Wachtler, Wiener med. Wochenschr. 1896.

5. Wintersteiner, Bericht über die deutsche ophthalmolog. Litteratur, II. Semester 1899. Zeitschr. f. Augenheilk. Bd. 4. S. 611.

6. Bondi, M., Zwei Fïlle einer in den Glaskürper vordringenden Arterien. schlinge. Klin. Monatsblātter f. Augenheill. 1899. S. 339.

7. Hirsch, C., Ein Fall von in den Glaskörper vordringender Gefässschlinge der Net\%hautschlagader. Klin. Monatsbliitter für Augenheilk. 1899. S. 341 .

S. v. Michel, Lehrbuch der Augenheilkunde. II. Aufl. 1890. S. 360 .

9. v. Hippel, Die Missbildungen und angeborenen liehler des $\lambda$ uges in Graefe-Sacmisch. II. Aufl. S. 72. Bd. II.

10. Licbreich, R., Transact. of the pathol. Society of London. V. 22. p. 222. (Cit. nach Günsburg.) 


$$
\text { Gräflin, Experimentelle Čntersuchungen etc. }
$$

11. Schapringer, ג., Knapp's Archiv für Augen- und Ohrenheilkunde. IlI.2. pag. 146. (S. a. Klin. Monatsblätter. 1599. S. 349.)

12. Becker, O., Krankheiten des Glaskörpers. Jahresbericht über die Leistungen und Fortschritte im Gebiete der Ophthalmologie. V. 1874. p. 500 .

13. S\%ili, Merkwürdige Schlinge der Netrhautschlagader. Centralblatt. 1885. S. 236.

IV.

(Aus der Basler Univ.-Augenklinik. [Prof. Mellinger]).

\section{Experimentelle Untersuchungen über den schädlichen Einfluss von pulverförmigen Anilinfarben auf die Schleimhaut des Kaninchenauges.}

Von

\section{I). ARTHURGRIFl,IN.}

Schon seit Jahren werden unserer lilinik und Poliklinik ron den hiesigen Falbfalniken Patienten zugeschickt, die sich infolge Eindringens von pulverförmigem liabstoff oder Farblösung in den Conjunctivalsack ane mehr oler weniger schwere Conjunctivitis und oft sogar auch Cornealerkrankung \%uge\%ogen hatten. Mit der Hebung der Farbindustrie, mit der Ausdchung ron Farbfilbriken und ihrer grüsseren Arbeiter\%ahl sind diese Frkrankungen häufiger geworden, und so haben dieselben in letrter \%eit auch das lnteresse der Ophthalmologen auf sich riehen miissen. IV ie wir uns bei einem Rundgang durch eine solehe Farbfilbrik über\%eugen kunnten, ist sowohl deren Direktion als aluch Arbeiterschatt der schädliche Einfluss einiger pulvertïrmiger liarbstoffe sowic Farbstofflösungen sehr wohl bekannt. Die Arbeiter haben daher Śchut\%l)rillen \%u tragen. Infolge Weglassens der Schut\%brille sind durch diese Farbstoffe dugenerkrankungen aufgetreten, die unsere Behandlung in Anspruch nahmen. Daneben siucl uns aber auch andere Patienten zugewiesen worden, die sich bei der Bearbeitung ron Farbstoffen, deren S̈chädlichkeit bis jetøt gar nicht bekimnt war, eine Iugenentründung \%uge\%ogen hatten. Cou sich üher die schäullichkeit oder Lnschädlichkeit einiger Anilinlarben \%u äber\%engen, haben wir deren Finfluss experimentell am Kaninchenauge exprobt.

Die bei den folgenden 1j Versuchen untersuchten Anilin- 